\title{
Cross Sectional Quantitative Survey in People for Disease Perception and Social Behaviour for Allergic Rhinitis
}

\author{
Article by Amrit Bhalchandra Karmarkar \\ PhD (Clinical Research) Scholar, Texila American University, South Guyana \\ E-mail: abkarmarkar@gmail.com
}

\begin{abstract}
The disorder with very common symptoms such as sneezing, itching, nasal congestion and rhinorrhea often remains undiagnosed in many individuals in the world. This is called as allergic rhinitis. According to WHO, this affects 10-30\% of world population. Prevalence of allergic rhinitis is around 7.8\% in USA, $5.9 \%$ in France and 29\% in United Kingdom (UK). However, in India no such study of prevalence was conducted in particular to study allergic rhinitis. Considering this fact, current study was aimed to determine disease perception and effect of allergic rhinitis on social behaviour in adult and elderly population in the areas of Mumbai metropolitan region in India using cross sectional survey methodology. Survey research of such type helps in gathering information not available from other sources. Disease perception and social behaviour assessments will help to understand psychosocial characteristics in population. Effect of allergic rhinitis on quality of life can also be investigated from this research. Questionnaire was designed and responses were collected by both online and offline modes. In total, 240 numbers of respondents agreed to participate. From the results, it was found that many respondents suffer from symptoms of sneezing, itching and nasal congestion which are cardinal symptoms of allergic rhinitis. From the results it was found that $66.67 \%$ of respondents have been diagnosed with allergic rhinitis from physician.
\end{abstract}

Keywords: allergic rhinitis, cross sectional survey, disease perception, social behaviour, India, clinical trials.

\section{Introduction}

The disorder with very common symptoms such as sneezing, itching, nasal congestion and rhinorrhea often remains undiagnosed in many individuals in the world. (Skoner DP, 2001). This is called as allergic rhinitis. World Allergy Organization (WAO) defines this disorder as a nasal disorder wherein an immune system produces response to allergen which is IgE mediated (WAO, 2016). WAO in depth review on allergic rhinitis suggests that around 400 million people suffer in the world from allergic rhinitis and most of the cases are undiagnosed and undertreated (Scarupa MD and Kaliner MA, 2015). According to World Health Organization (WHO), this affects 10-30\% of world population (WHO, 2011). Prevalence of allergic rhinitis is around 7.8\% in United States of America (USA), $5.9 \%$ in France and 29\% in United Kingdom (UK). However, in India no such study of prevalence was conducted in particular to study allergic rhinitis. Unofficially, it was suggested by researchers that approximately, 20-30\% of Indians suffer from at least one allergic disease (Prasad R and Kumar R, 2013). Only data from study conducted in year 1964 suggests that prevalence of allergic rhinitis was $10 \%$ in India (Viswanathan R, 1964). Allergic rhinitis and its impact on asthma (ARIA) initiative in its Asia Pacific workshop report highlights the fact that this disorder has not received attention of both physicians and patients (Shah and Pawankar, 2009).

Most of the patients of allergic rhinitis often ignore this order as they feel it as common cold and their symptoms. However, it was observed that allergic rhinitis reduces quality of life (QOL) significantly. In particular, it affects social behavior of patient (Scadding et al. 2008). Therefore, it is decided to estimate disease perception and effect of allergic rhinitis on social behaviour using cross sectional survey in adult and elderly population. Children will not be included in survey because their inability to understand symptoms, pathology and treatment of disorders. 


\section{Methods}

As emphasized above, Survey research of such type helps in gathering information not available from other sources (Grill JD et al., 2015; Smith SK et al., 2015; and Marcano Belisario JS et al., 2015). Disease perception and social behaviour assessments will help to understand psychosocial characteristics in population. Effect of allergic rhinitis on quality of life can also be investigated from this research. Before making study protocol, pre-pilot or exploratory study and pilot study were carried out.

\section{Pre-pilot study}

Pre-pilot study serves as thought clarifying stage for qualitative research. This involved conversation with one female ENT physician with 25 years' experience. This conversation serves as essential step in design of questionnaire for protocol.

\section{Preparation of draft of protocol for cross sectional quantitative survey}

After pre-pilot study, relevant literature search was done and draft protocol of quantitative cross sectional survey was written. Protocol contained outline such as introduction, study design, brief information of disease, and 18 multiple choice questions. This protocol was then subjected to pilot study.

\section{Pilot study}

Draft of protocol of cross sectional quantitative survey was sent to Dr. Indrajeet Gonjari, Research Guide, Texila American University, South Guyana and also discussed with one MD; two MBBS; one MS (ENT).

\section{Finalisation of protocol for cross sectional quantitative survey}

Changes suggested by all of them were made and protocol was finalised.

\section{Summary of study design}

This study is prospective observational (quantitative cross sectional questionnaire based survey evaluation) study wherein common public or general population will enrolled as participants. Participants were be provided with questionnaire on allergic rhinitis. Participants have to select one option from multiple choices provided in that question. Quantitative evaluation of responses was done via data analysis.

\section{Purpose}

Purpose of present study is to determine disease perception and social behaviour in general population with cross sectional quantitative survey method.

\section{Study population}

General population or common public with age above 18 years can participate in study.

Both male and female were allowed to take part in study.

\section{Study setting}

Study was carried out in adult and elderly population in Mumbai metropolitan region of India using offline and online questionnaires.

\section{Inclusion criteria}

Participant (i.e. general population / public) must satisfy following requirements:

- $\quad$ Age 18 years and above

- Gender: Both male and female

- No history of psychotic condition 


\section{Exclusion criteria}

Participant should not have

- $\quad$ Age below 18 and above 70

- Any psychotic conditions

\section{Study population and sample size}

Study involved use of questionnaire based survey of adult and elderly population. Children were excluded due to lack of their knowledge about symptoms. Sample size of around 100 was expected.

\section{Study duration}

Study was conducted from 01 November 2016 to 15 March 2017.

\section{Ethical considerations}

As this is non-interventional study in general population, no ethical approval was obtained. Confirmation of regarding approval was obtained from board of studies of Texila American University, South Guyana.

\section{Participant recruitment or advertising}

Participants were approached directly and also using social media platforms such as Facebook, Twitter, WhatsApp, and website postings to get greater number of responses to questionnaire.

\section{Outcome measures}

Following outcome measures will be identified from questionnaire.

- Disease perception

- Social behaviour

- Awareness of symptoms

- Diagnosis, and treatment patterns

\section{Recording, data collection and analysis}

Questionnaire responses were collected via both offline and online modes. Data was entered in Microsoft Excel 2007 and data was represented graphically in form of bar graphs. Wherever applicable, percentage analysis will be done. Data analysis and representation will be done using Microsoft Excel 2007.

\section{Results}

Survey research of such type helps in gathering information not available from other sources (Grill JD et al., 2015; Smith SK et al., 2015; and Marcano Belisario JS et al., 2015). From the current research, following observations were obtained.

\section{Response rate}

As mentioned in methods, current research was carried out in both online and offline ways. In total, 350 respondents were approached. Out of them, 240 respondents actually replied to the survey. In total, response rate was found to be $68.57 \%$. Out of total respondents, $58.33 \%$ respondents were participated using online mode and $41.67 \%$ were from offline mode.

\section{Gender and mode of response of survey}

In this survey, participants have taken part in both online and offline mode. Figure 2 represents this data graphically. 
DOI: $10.21522 / \mathrm{TIJCR} .2014 .04 .02 . A r t 004$

ISSN: 2520-3096

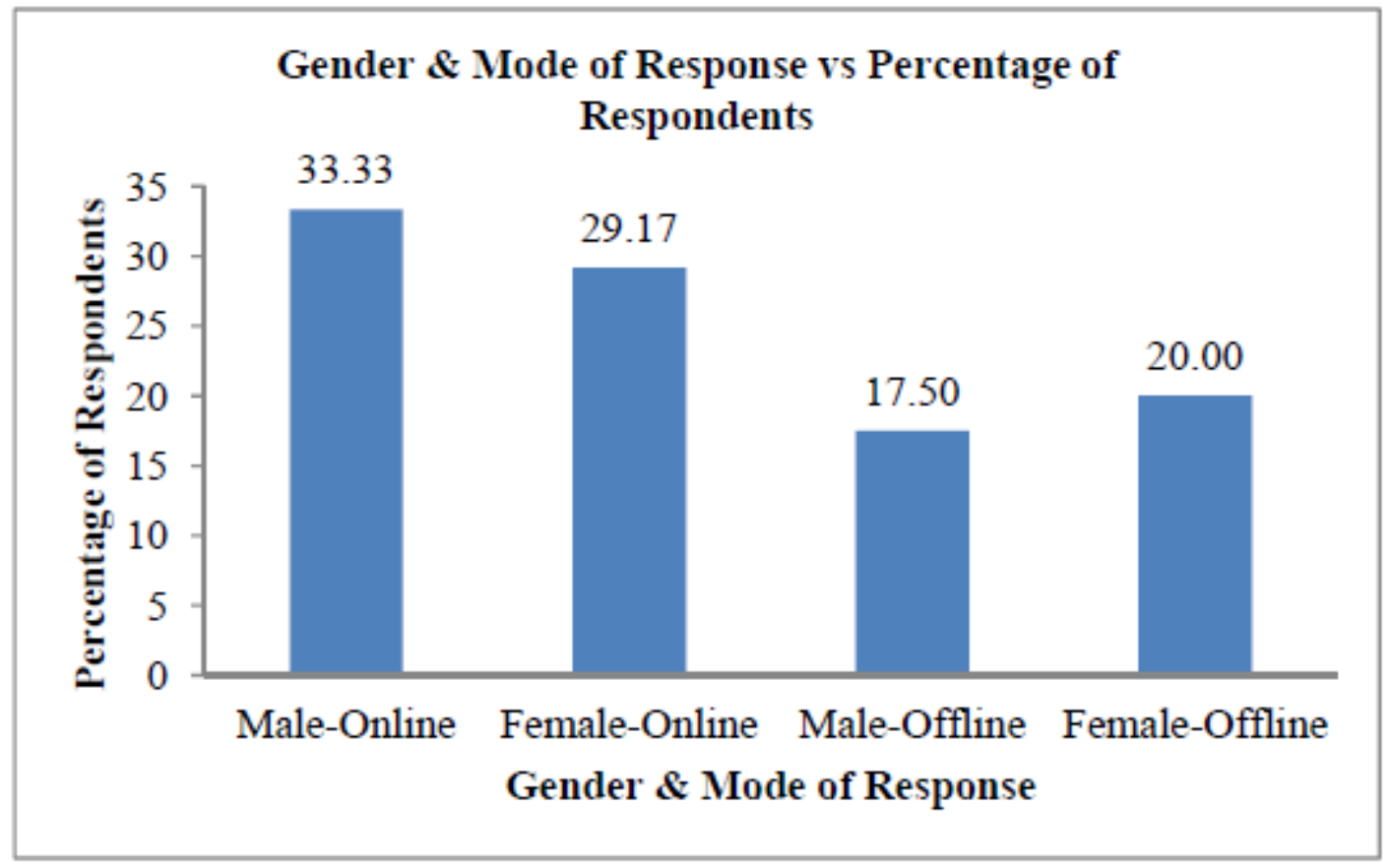

Figure 1. Gender and mode of response vs. percentage of respondents

\section{Average travel time for work}

As travel time for work is most important factor in Mumbai, it has taken into consideration. During travel, different mode of transport i.e. rail, and road are used daily by people. Certainly this may have impact on causation of allergic rhinitis. Table 1 highlights this fact.

Table 1. Average travelling time for work

\begin{tabular}{|l|l|}
\hline Travelling time for work & Percentage of Respondents \\
\hline 0 to 0.5 hour & 6.25 \\
\hline 0.5 to 1 hour & 38.33 \\
\hline 1 to 1.5 hour & 34.58 \\
\hline 1.5 to 2.0 hour & 15.83 \\
\hline 2.0 or more & 5.00 \\
\hline
\end{tabular}

\section{Response about different nasal symptoms}

Participants were asked about whether they have any of such symptoms such as symptoms on one side of nose; thick, green or yellow discharge from nose; post nasal drip (down back of the throat) with thick mucus and/or runny nose; facial pain; recurrent nosebleeds; and loss of smell. Figure 2 graphically represents the data. 


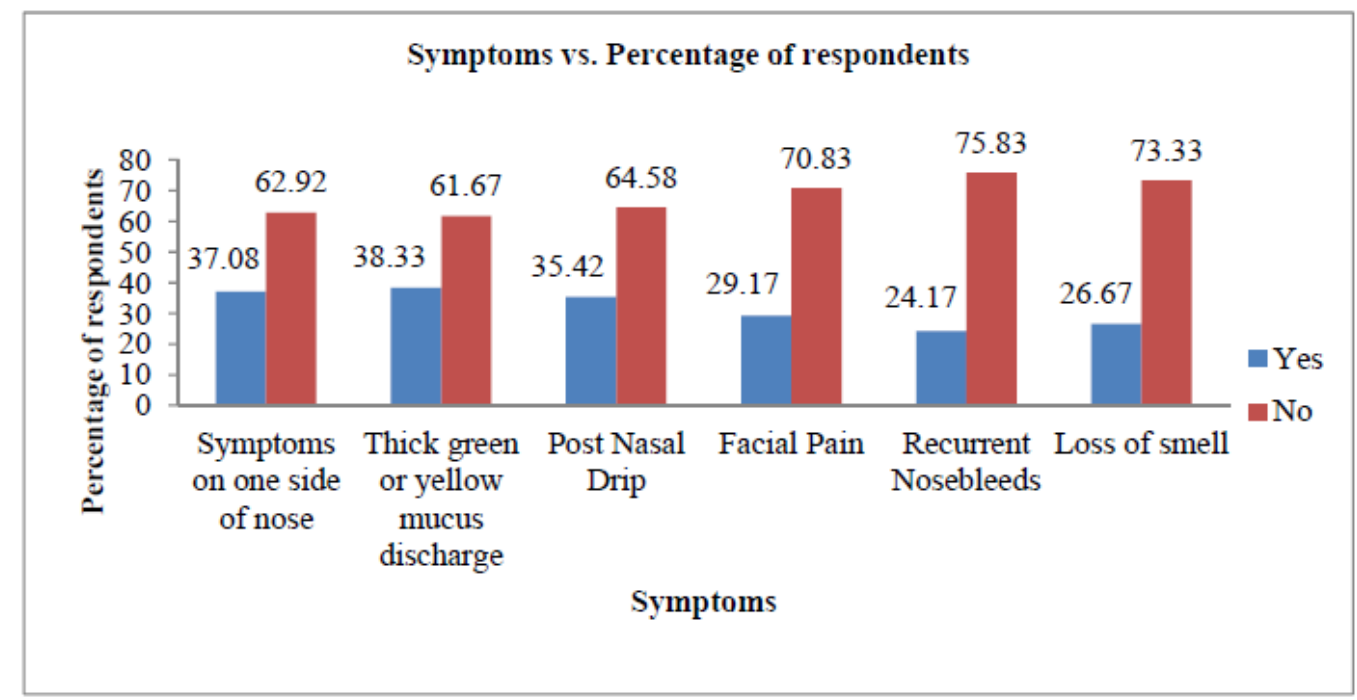

Figure 2. Symptoms vs. percentage of respondents

\section{Symptoms lasting at least one hour on most days}

Participants were asked about whether they experience symptoms such as watery runny nose, sneezing especially violent and in bouts, nasal obstruction, nasal itching, conjunctivitis (red, itchy eyes) for at least one hour on most days, or during season if their symptoms are seasonal. Following responses were obtained and are represented graphically in Figure 3.

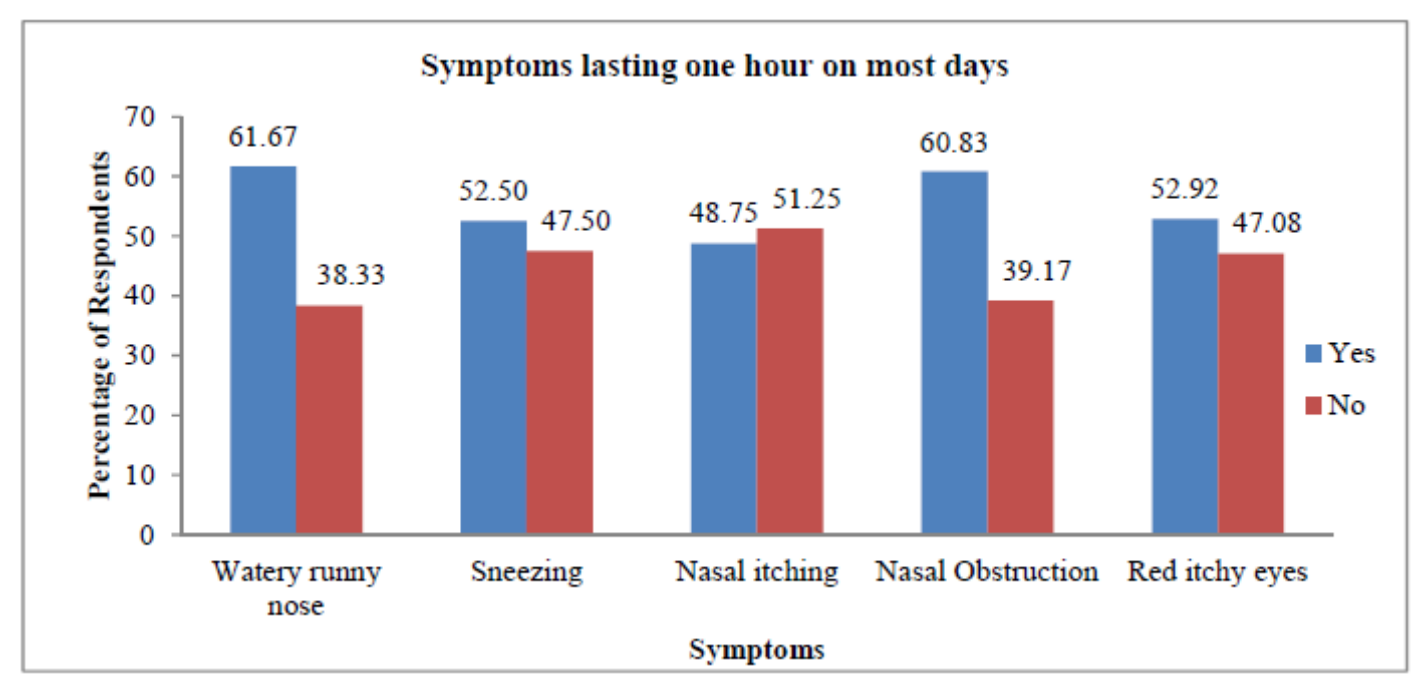

Figure 3. Symptoms lasting at least one hour on most days

\section{Grade of troublesome of symptoms}

Participants were asked how troublesome these symptoms are to them. Following responses were obtained. 
DOI: $10.21522 /$ TIJCR.2014.04.02.Art004

ISSN: 2520-3096

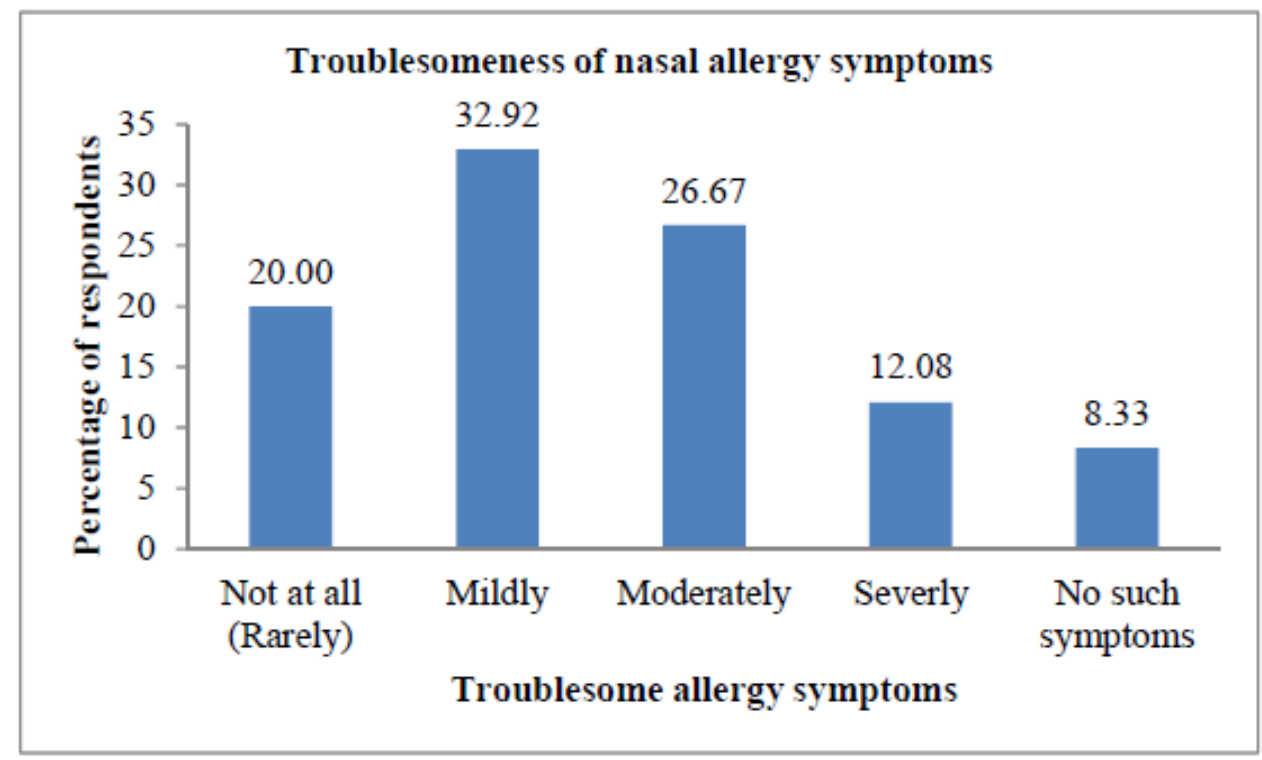

Figure 4. Troublesome allergy symptoms

\section{Causes of symptoms}

According to literature, different causes of allergic rhinitis were studied (Scarupa MD, \& Kaliner MA, 2015). These include pollen from trees, flowers and grasses; mould (both indoor and outdoor), furred animals (especially cats, dogs and mice), dusty places, and air pollution. Following responses were outlined in Figure 5.

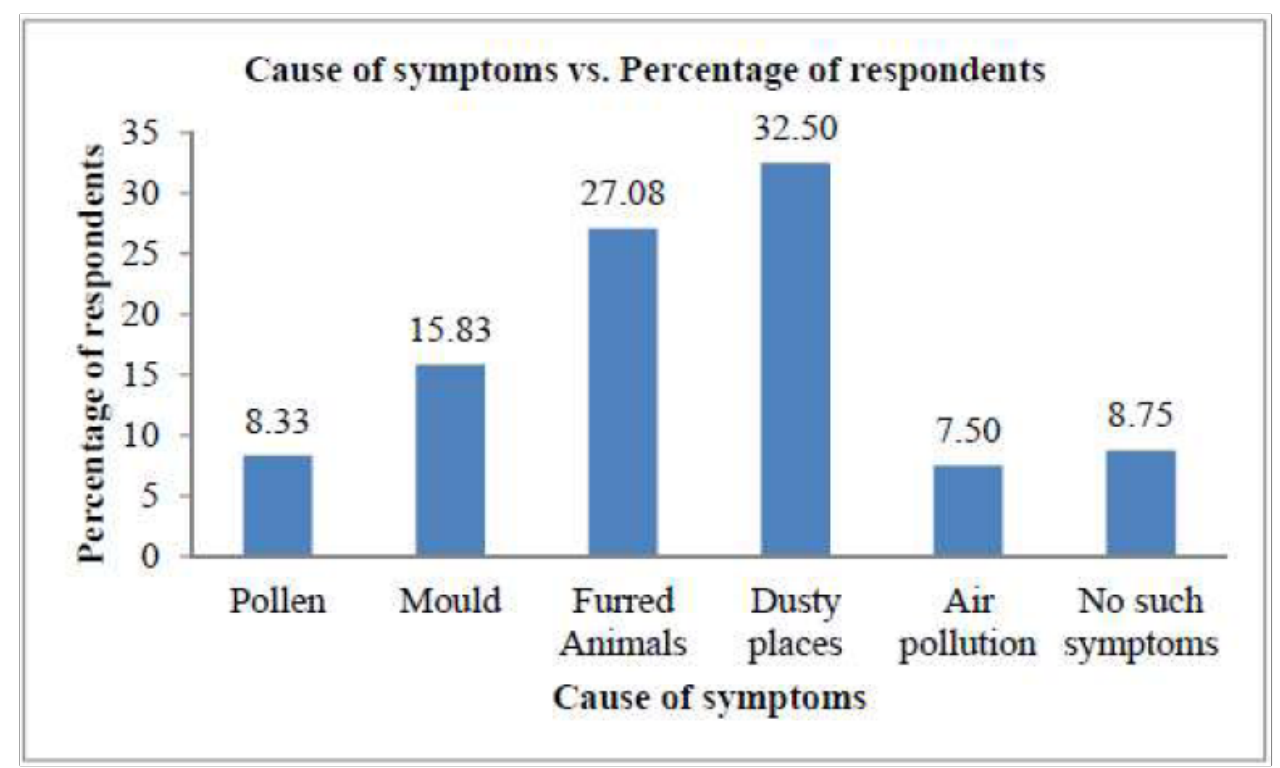

Figure 5. Cause of symptoms vs. percentage of respondents

\section{Effect on activities of respondents}

Question was asked to respondents about how symptoms affect their activities. This included disturbance in sleep, restriction in daily activities, and restriction in participation in school or work. Following responses were obtained and are represented in Figure 6. 


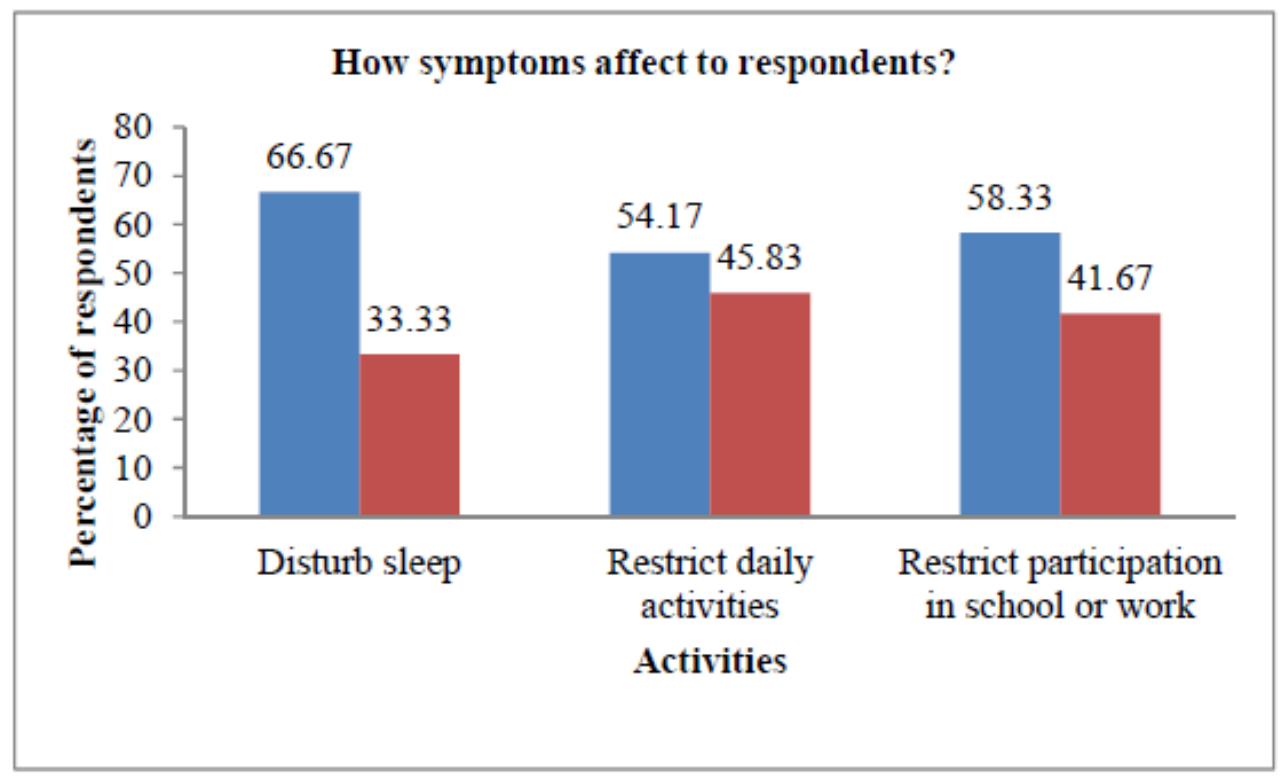

Figure 6.Effect of symptoms on activities of respondents

\section{Duration of symptoms and its effect on meeting with people}

Participants were asked about how long these symptoms last and do they have effect on meeting with people. Following observations were obtained and are represented graphically in Figure 7 and 8.

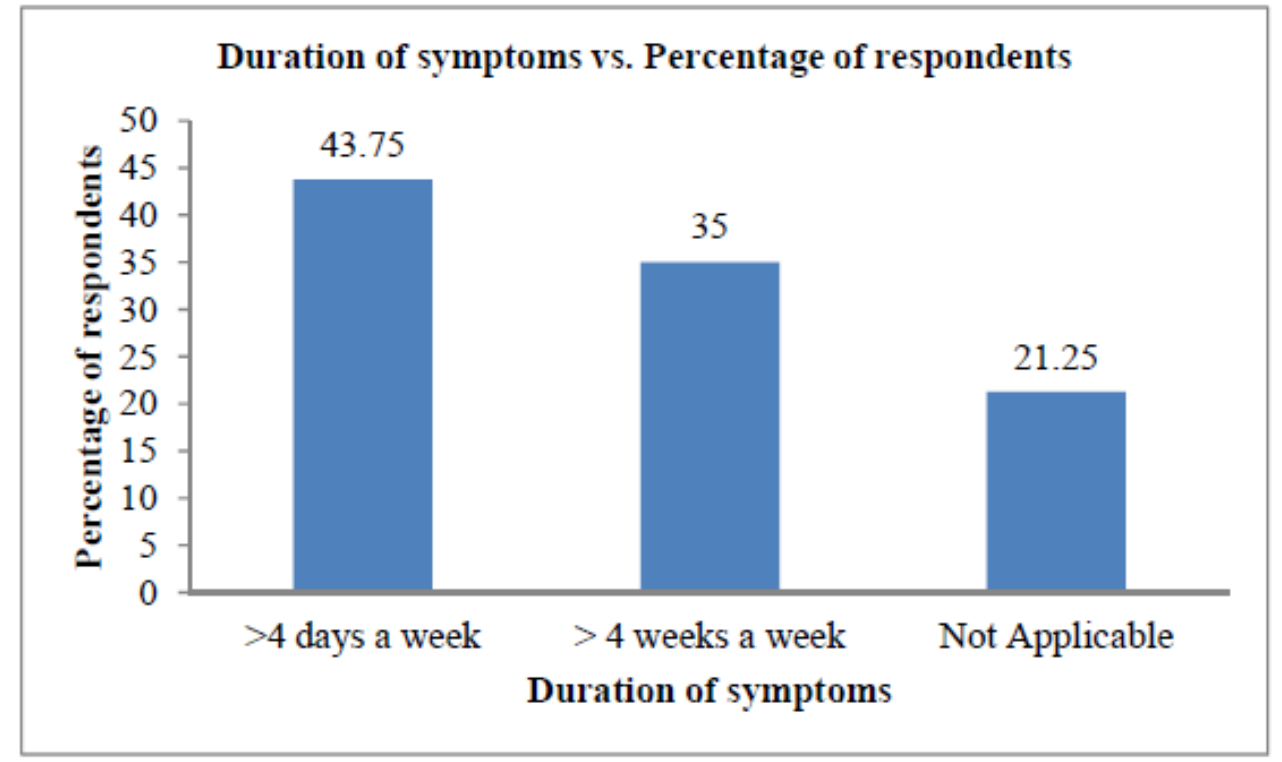

Figure 6. Duration of symptoms 
DOI: $10.21522 /$ TIJCR.2014.04.02.Art004

ISSN: $2520-3096$

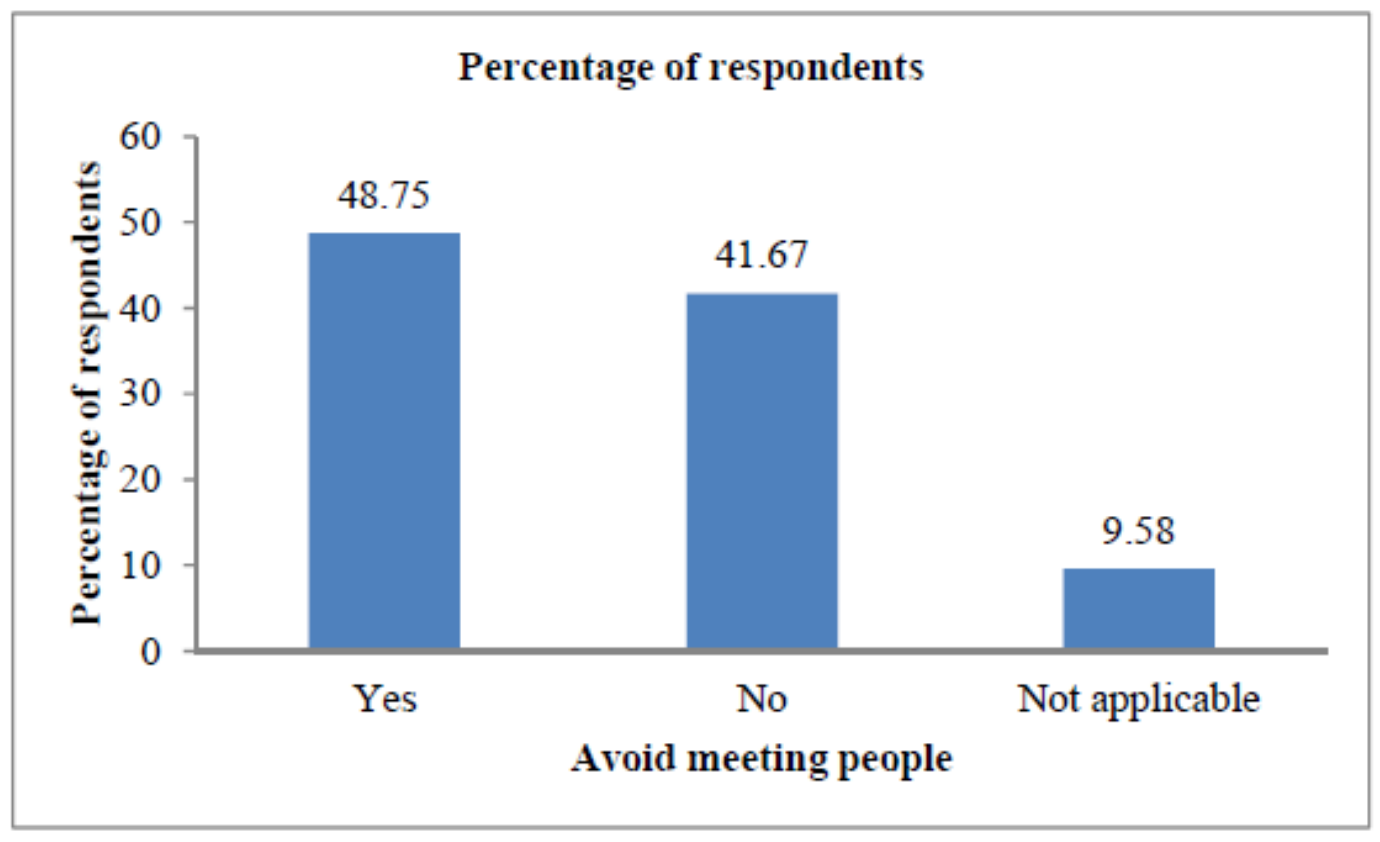

Figure 7. Response of respondents that avoid meeting with people due to symptoms

From the figure 7 it could be said that around $48.75 \%$ respondents avoid meeting with people due to nasal and other symptoms.

\section{Effect on quality of life}

Respondents were asked about how allergic rhinitis symptoms have affected their quality of life. Figure 8 graphically represents the data.

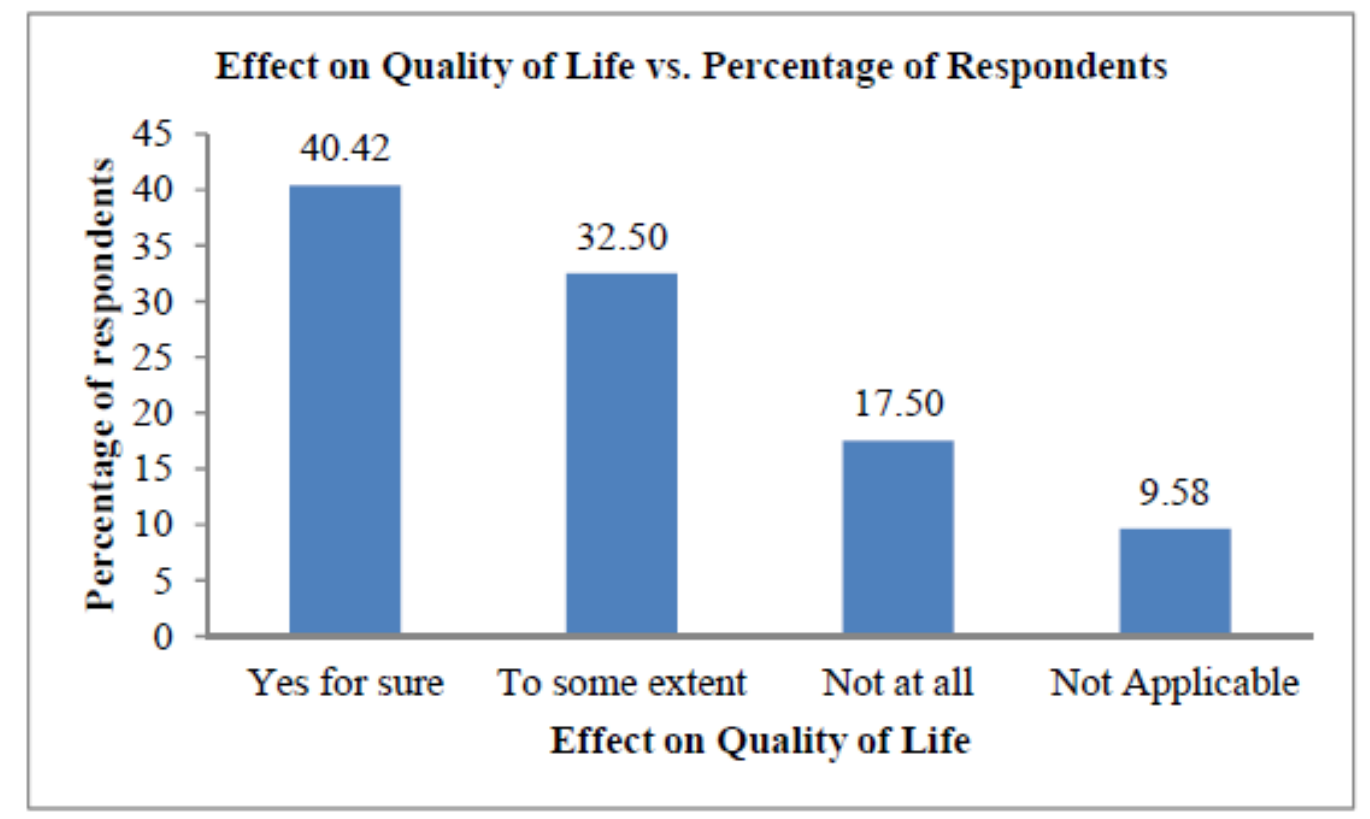

Figure 8. Effect on quality of life

\section{Effect on mood}

Question was asked to participants about whether they get irritated, depressed easily or little or not at all due to symptoms of allergic rhinitis. About $27.92 \%$ replied that they get irritated easily, and $25.42 \%$ replied that they get depressed easily. Figure 9 represents the data. 


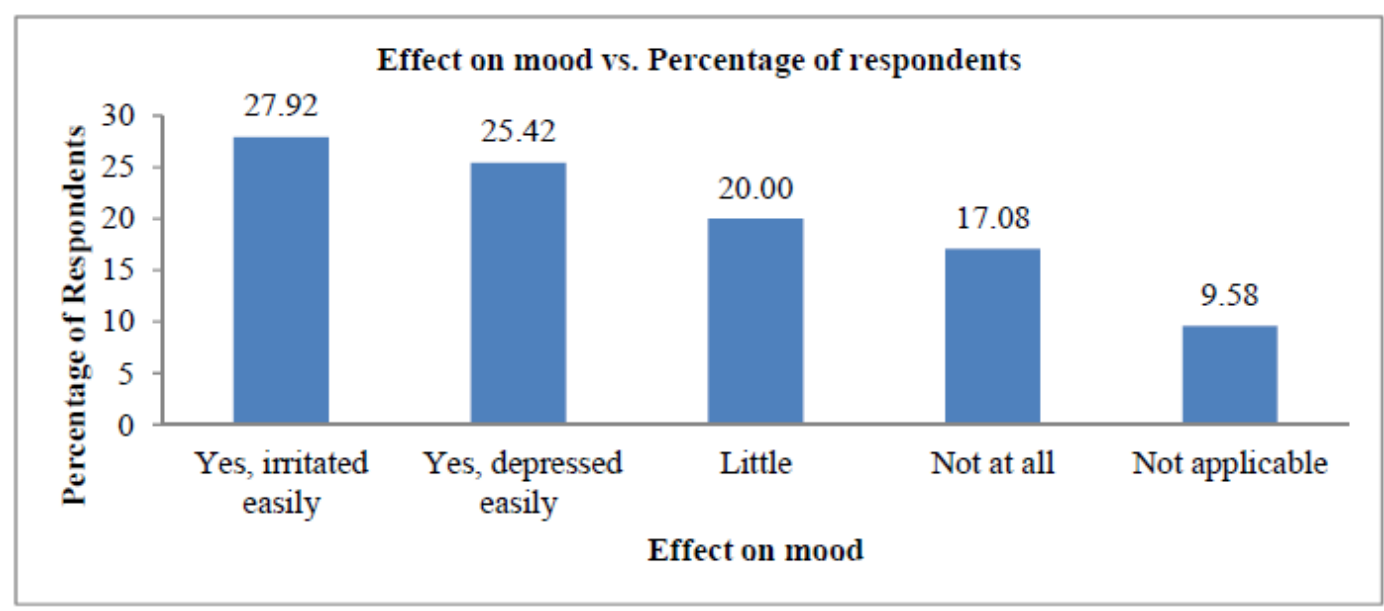

Figure 9. Effect on mood due to symptoms

\section{Effect on relations}

Question was asked to participants about how symptoms affect to their work and private life. Following responses were obtained and are shown in Figure 10.

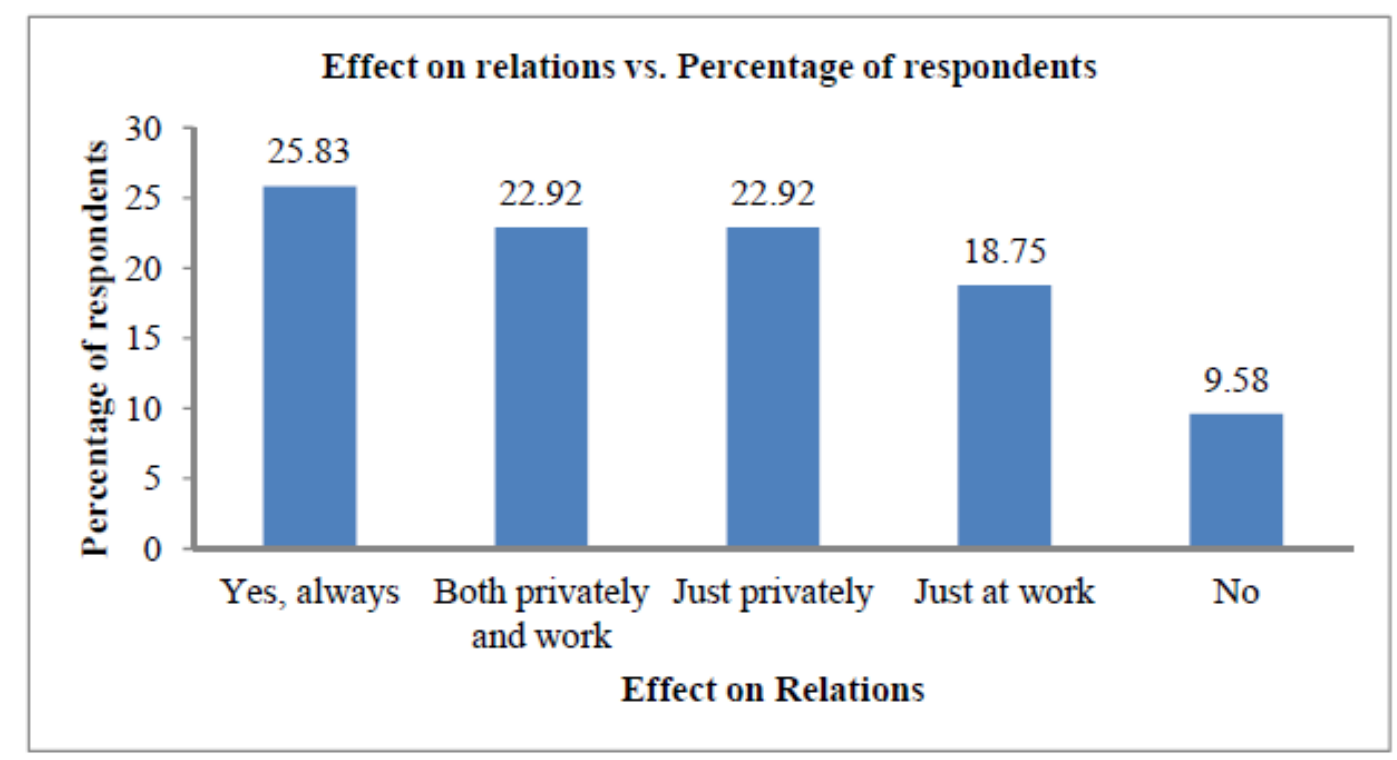

Figure 10. Effect of symptoms on relations

\section{Explanation of allergic rhinitis}

Question was based on how participants explain allergic rhinitis. Whether they explain it to others in detail, or does not explain or avoid it or even don't know how to explain. This was represented as follows in Figure 11. 
DOI: $10.21522 /$ TIJCR.2014.04.02.Art004

ISSN: $2520-3096$

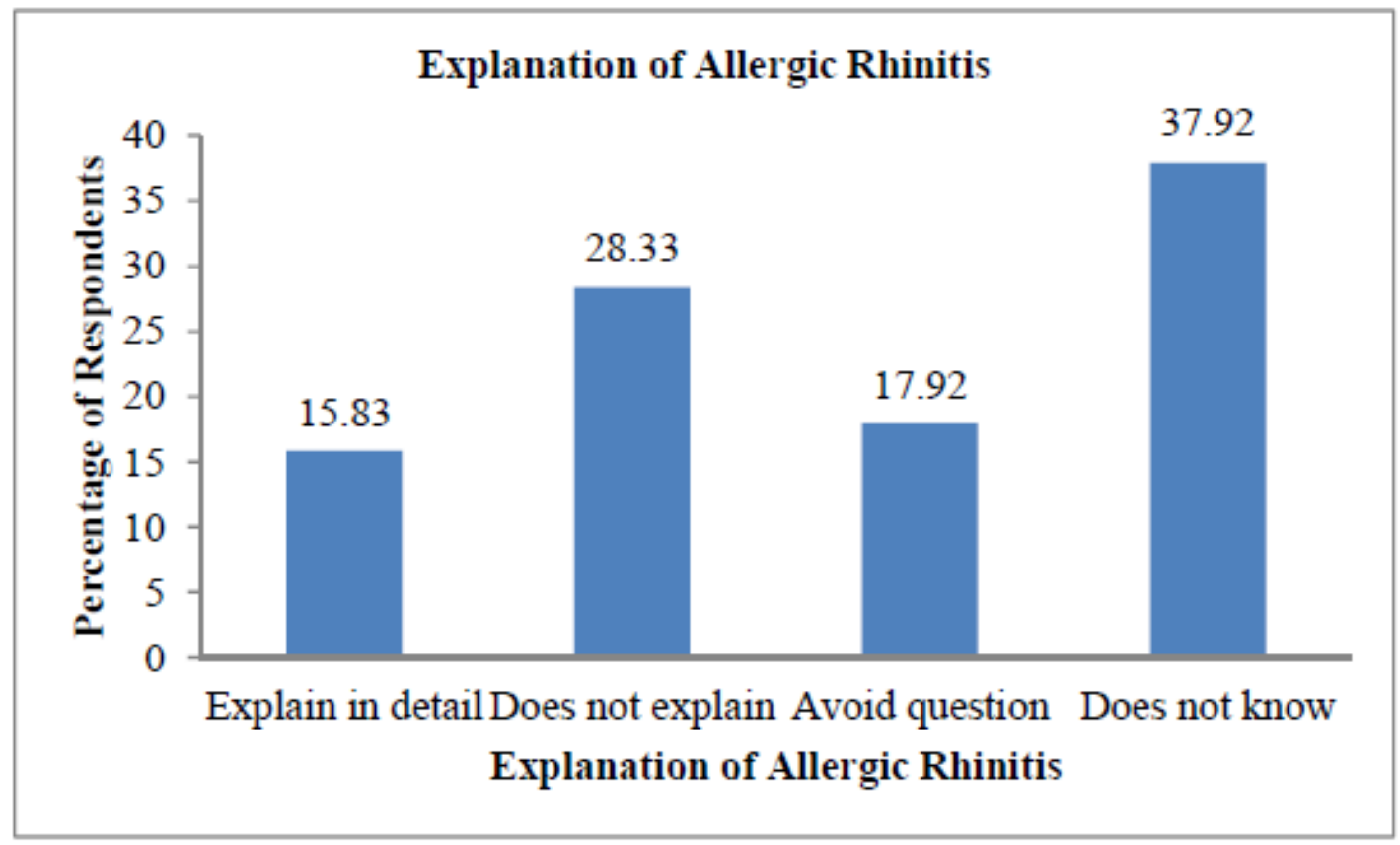

Figure 11. Explanation of allergic rhinitis

\section{Allergy testing and type of allergy testing}

Participants were asked about whether they have done allergy testing. Only $15.83 \%$ of participants responded that they have done allergy testing. Further, what type of allergy testing was done was asked to respondents who replied yes to above question, following results were obtained.

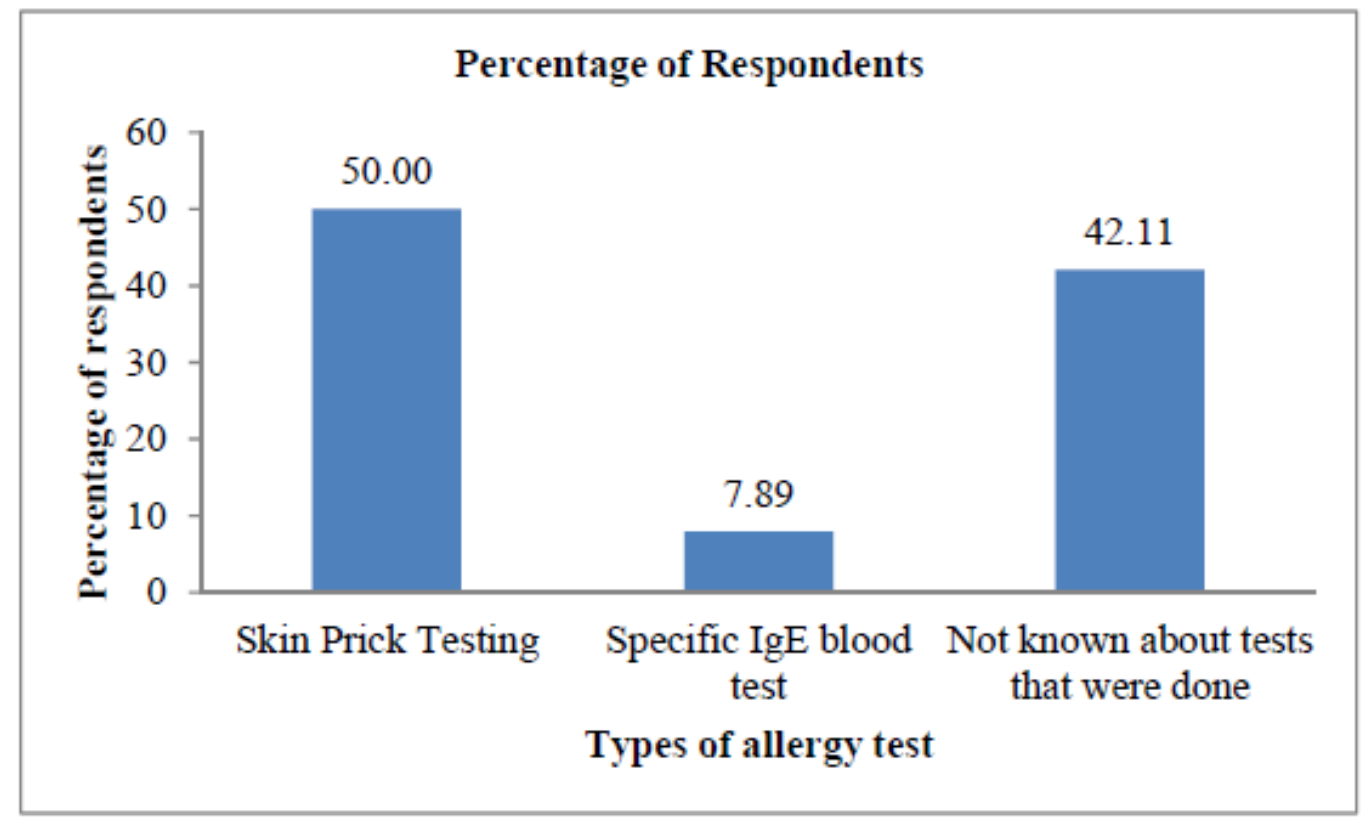

Figure 12. Type of allergy test done by respondents

\section{Family member with allergy}

Around $72.92 \%$ of respondents replied that they have some of the family members who have allergic rhinitis or some other allergic diseases. $27.08 \%$ replied that they do not have allergic family member. 


\section{Diagnosis of allergic rhinitis}

Out of total respondents, $66.67 \%$ of respondents replied that they have been diagnosed with allergic rhinitis and $33.33 \%$ said they have not been diagnosed with disease.

\section{Type of physician from which treatment was sought}

Question was asked about from which type of physician they have received treatment. In India, MBBS and MD (Allopathic); BAMS and MD (Ayurvedic); BHMS and MD (Homeopathic); BUMS and MD (Unani) systems of medicine are generally available to population. From the results, it is suggested that allopathic treatment option is most preferred amongst population.

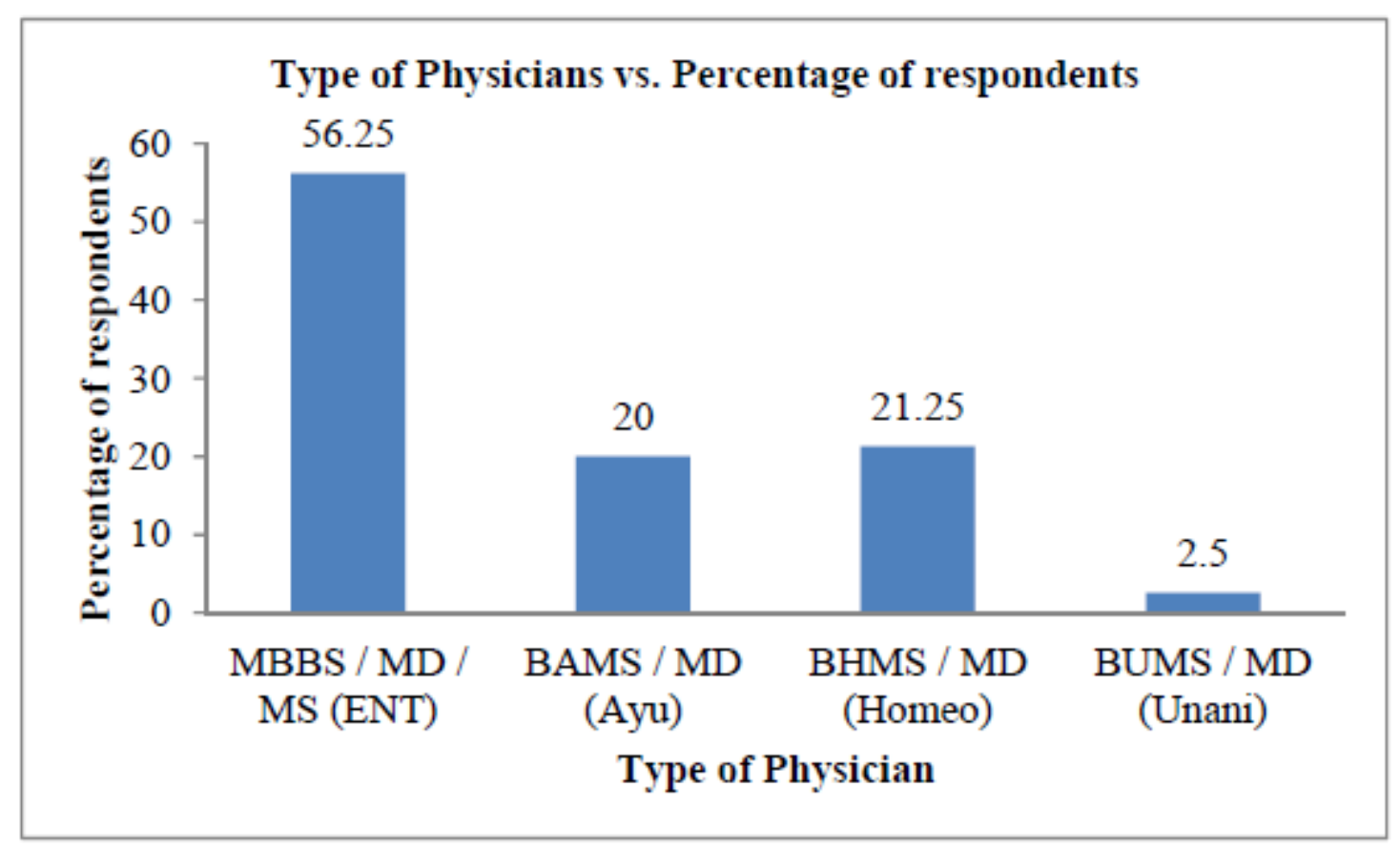

Figure 13. Type of physician from which treatment is taken

\section{Type of treatment}

Regular medicine, symptomatic medicine and combination of two therapies are options for treatment of allergic rhinitis treatment. In around $53.13 \%$ of respondents regular therapy was provided. Figure 14 shows data of these results. 
DOI: $10.21522 / \mathrm{TIJCR} .2014 .04 .02 . A r t 004$

ISSN: 2520-3096

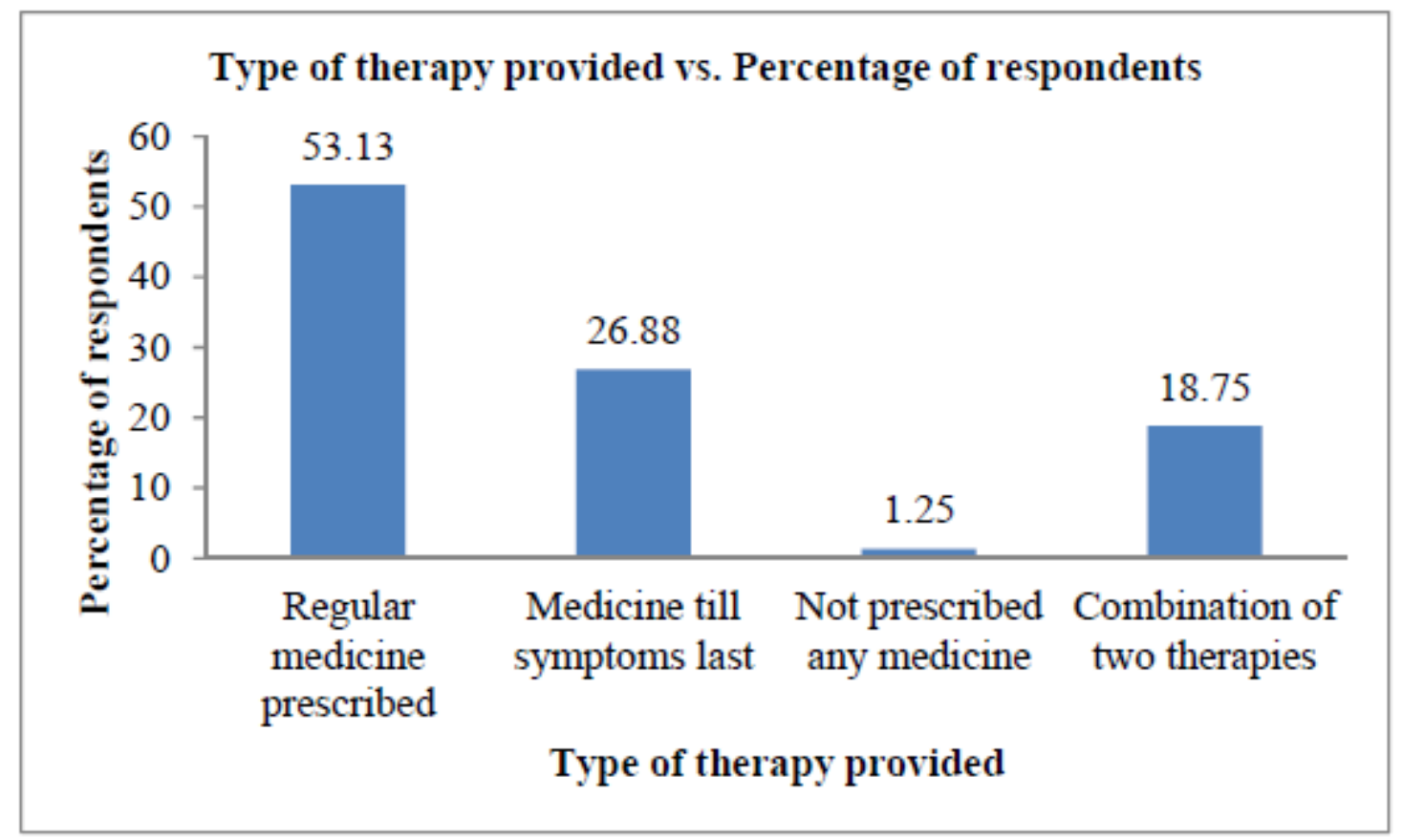

Figure 14. Type of therapy provided for allergic rhinitis

\section{Effectiveness of therapy and choice of second opinion}

Participants were asked about how effective therapy was there for allergic rhinitis. Data from results is shown in Figure 15.

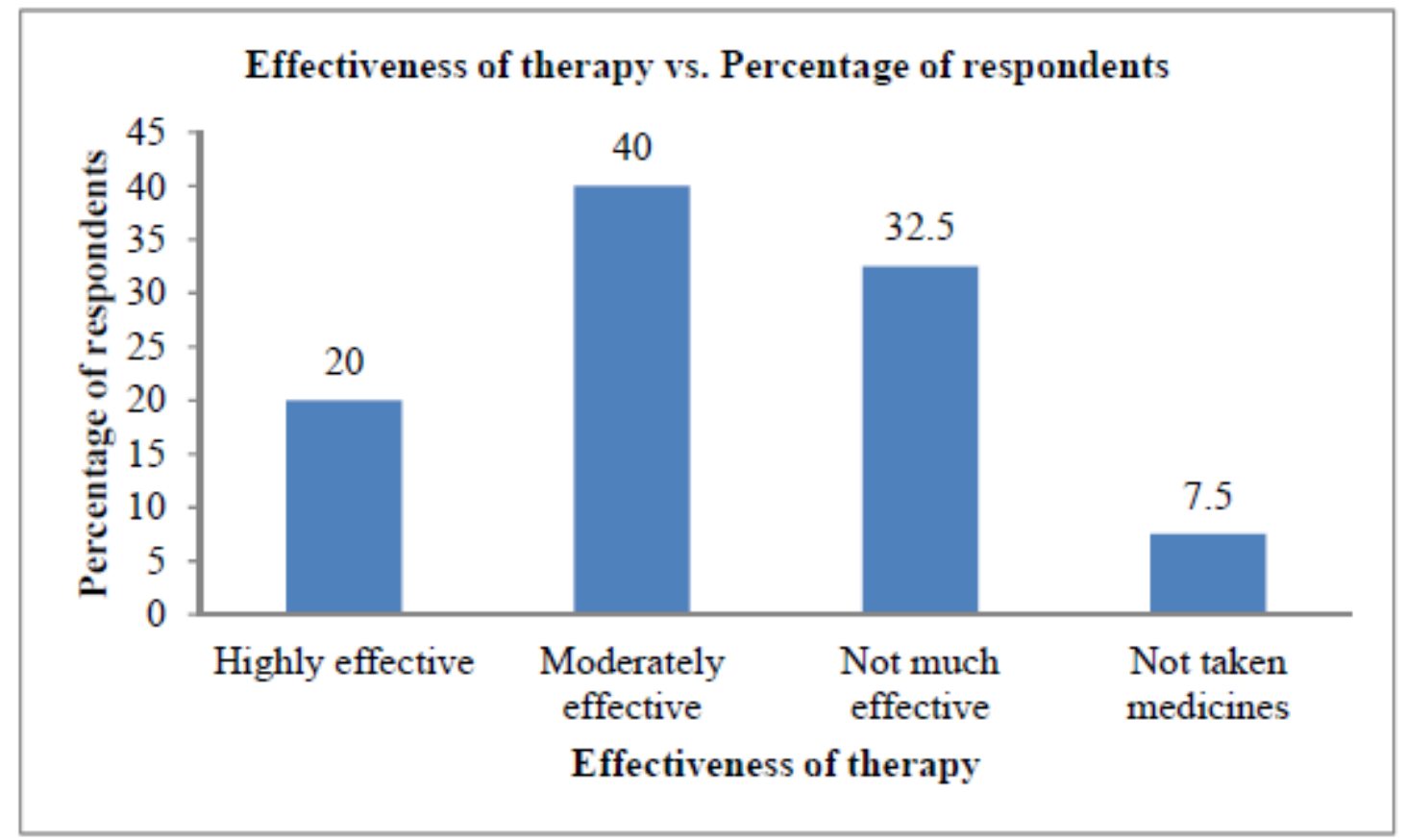

Figure 15. Effectiveness of therapy for allergic rhinitis

They were also asked about whether they opted for second opinion from any other physician. Only $13.75 \%$ respondents have taken second opinion and $86.25 \%$ respondents have not opted for second opinion. 


\section{Discussion}

Current study was aimed to determine disease perception and effect of allergic rhinitis on social behaviour in adult and elderly population in the areas of Mumbai metropolitan region in India using cross sectional survey methodology. Survey research of such type helps in gathering information not available from other sources. Disease perception and social behaviour assessments will help to understand psychosocial characteristics in population. Effect of allergic rhinitis on quality of life can also be investigated from this research.

Questionnaire was designed and responses were collected by both online and offline modes. In total, 240 numbers of respondents agreed to participate. Out of 350 respondents who were approached, only 240 actually participated. This means that only $68.57 \%$ responded the survey. According to Jack Fincham (2008), goal of the study should be collection of at least $60 \%$ of responses. For survey based research, $\geq 80 \%$ should be there to appropriately represent population. Reaching only $68.57 \%$ is limitation of the current research. This may be due to limited study time period and unawareness of allergies and allergic diseases in population (Nulty DD, 2008).

Travelling with allergies is difficult and travelling incurs exposure with allergens such as pollens, air pollution, etc. In Mumbai, average travelling time for 38.33\% of respondents was up to 1 hour and for $34.33 \%$ was up to 1.5 hours. Blum SW et al (2015) have suggested that travelling time increases burden of allergic rhinitis in United States.

Mode for conduction of this research were both online and offline surveys. Offline or paper based surveys provide original material related to survey methods, and also we can get number of recommendations from the respondents additionally. However, targeting participants by visiting their home or office becomes a tedious task and also it increases cost of conduction of the study. In case of online surveys, the convenience of response can be achieved and survey link can be easily sent to respondent's smart phone or email id (Evans JR and Mathur A, 2005). Due to this fact, response of online questionnaire was found more than offline in current survey. This fact is also highlighted when gender based filter is applied to responses of the study. Both males and females preferred online mode more than paper based survey.

Symptoms on one side of nose, thick green or yellow discharge of mucus, post nasal drip, facial pain, recurrent nose bleeds, and loss of smell are generally not found in Allergic rhinitis (Refer Figure 2). Presences of any one of these symptoms indicate alternative diagnosis and referral of specialist is recommended. Results of the study indicate that most respondents are not experiencing these symptoms. Out of these symptoms, facial pain, loss of smell, and post nasal drip are seen in sinusitis. In this case, specialist should be consulted. However, any one of symptoms such as watery runny nose, sneezing, nasal itching and obstruction, and conjunctivitis (including red itchy eyes) indicate possibility of allergic rhinitis. Findings of the study (Figure 3) indicated that most of participants (50$60 \%$ ) are having possibility of allergic rhinitis. These findings are in accordance with guideline of Allergic Rhinitis and its impact on Asthma (ARIA) (2007).

Question was asked to participants about how troublesome these symptoms are. According to results, $32.92 \%$ reported it to as mild, $26.67 \%$ respondents reported it is moderately troublesome and $12.08 \%$ reported it as severe. This indicates allergic rhinitis to them. These findings are in accordance with statements made by Wallis RS (1982).

Increase in allergen exposure and reduced immunity are important factors from which allergic rhinitis could occur. Exposure to allergens such as pollen, moulds, furred animals, dust, air pollution are some these allergens. (Mandhane SN et al. 2011).

It was found that for participants where symptoms were there, disturb in sleep, daily activities, and participation in school work were hampered. In most of the respondents, symptoms were found to last for more than 4 days a week. This had impact on meeting with people. These findings are in accordance with ARIA guidelines. This indicates persistent form of allergic rhinitis. (Bachert et al, 2002). "A subjective value a person places on satisfaction with own life is called as Quality of Life". Figure 8 indicates that allergic rhinitis affects quality of life of an individual. This finding is in accordance with findings of Spanish study which suggests that allergic rhinitis affects quality of life more than that of diabetes, hypertension and depression (de la Hoz Caballer B, 2012). 
Marshall PS et al (2002) have evaluated effect of allergic rhinitis due to pollens and found that fatigue and alterations in mood occur due to it. According to him, this could be change in biochemical reactions in central nervous systems due to which mood changes occur. Sansone RA and Sansone LA (2011) have evaluated relationship of allergic rhinitis on mood and anxiety syndromes. Results of current research are in accordance with these studies. In total $73.33 \%$ (Figure 9) (i.e. total of $27.92 \%$ who get irritated easily, $25.42 \%$ who get depressed, and $20.00 \%$ who have little effect on mood levels) of respondents witnessed changes in mood due to allergic rhinitis. Similar effect was observed with relationships of allergic rhinitis respondents (Figure 10). Also in this study, participants were asked about how they explain symptoms of disease. Most of the respondents, however, do not know how to explain symptoms to others. This suggests need of awareness programs on allergic rhinitis.

In total, $84.17 \%$ respondents have not done allergy testing. Out of those who did allergy testing, $50.00 \%$ have done with skin prick testing, $7.89 \%$ with specific IgE testing, and $42.11 \%$ are not aware of which testing was done. Therefore, this suggests need for awareness of allergic testing which is integral part of diagnosis and treatment. Similar facts were suggested by Kalpakliglu AF et al (2011).

Around $72.92 \%$ of respondents said that their family member has allergic rhinitis. Genetic segregation studies and investigations in twins have suggested genetic basis of causation of allergic rhinitis. Studies on chromosome 2, 3, 4, and 9 revealed the fact of genetic hereditary link between allergic rhinitis (Davila I, 2009). De Yun Wang (2005) has also highlighted the same fact.

It was found that $66.67 \%$ respondents have diagnosed with allergic rhinitis. In India, different options for treatment are available. Allopathic treatment is most preferred and then ayurvedic and homeopathic therapies are preferred by respondents. Regular medicine, symptomatic medicine and combination of two therapies are options for treatment of allergic rhinitis treatment. In around 53.13\% of respondents regular therapy was provided. Figure 14 shows data of these results. According to ARIA guidelines, effectiveness of treatment of allergic rhinitis is very less. Results from Figure 15 represent the data (Mandhane SN, 2011). Seeking second opinion to other physician is the other way to confirm the diagnosis and treatment of disease. In the current research, only $13.75 \%$ respondents have taken second opinion and $86.25 \%$ respondents have not opted for second opinion. Gendo and Larson (2014) used method of second opinion to confirm the diagnosis of suspected allergic rhinitis.

\section{Conclusion}

Disease perception and social behaviour assessments helped in gathering data about allergic rhinitis. Parameters such as nature of symptoms, troublesome behaviour of symptoms, cause of symptoms, how they affect daily activities and how long they last, effect on meeting with people and quality of life, effect on mood and relationships were investigated. Also other factors such as allergy testing, type of allergy testing, diagnosis, treatment, duration and effectiveness of treatment were studies. Thus, survey type of research helped in gathering information not available from other sources, especially about psychosocial characteristics in population. From the results it can be concluded that prevalence of allergic rhinitis is significant in population. However, awareness and treatment strategies need to be improved. Further research will be conducted on opinions of physicians about treatment pattern and awareness about allergic rhinitis in population.

\section{References}

[1].Bachert C, van CauwenbergeP, Khaltaev N (2002); World Health Organization. Allergic rhinitis and its impact on asthma. In collaboration with the World Health Organization. Executive summary of the workshop report. Geneva, Switzerland, December 7-10, 1999. Allergy; 57:841-55.

[2].Blume SW, Yeomans K, Allen-Ramey F, et al (2015). Administration and Burden of Subcutaneous Immunotherapy for Allergic Rhinitis in U.S. and Canadian Clinical Practice. J Manag Care Spec Pharm. 21(11); 982-990.

[3].Davila I, Mullol J, Ferror M, et al. (2009) Genetic aspects of allergic rhinitis. J Investig Allergol Clin Immunol. 19(1); 25-31.

[4].de la Hoz Caballer B, Rodriguez M, Fraj J, et al (2012). Allergic rhinitis and its impact on work productivity in primary care practice and a comparison with other common diseases: the Cross-sectional study to evaluate 
work productivity in allergicrhinitis compared with other common diseases (CAPRI) study. Am J Rhinol Allergy; 26:390-4.

[5].De Yun Wang (2005). Risk factors of allergic rhinitis: genetic or environmental? Ther Clin Risk Management. 1(2); 115-123.

[6].Evans JR, Mathur A (2005). The value of online surveys. Internet Research. 15 (2); 195-219.

[7].Fincham JE (2008). Response Rates and Responsiveness for Surveys, Standards, and the Journal. Am J Pharm Edu. 72(2); 43.

[8].Gendo K, Larson EB (2014). Evidence-Based Diagnostic Strategies for Evaluating Suspected Allergic Rhinitis. Ann Int Med. 140; 278-289.

[9].Grill JD, Bateman RJ, \& Buckles VA (2015). Survey of attitudes toward clinical trials and genetic disclosure in autosomal dominant Alzheimer's disease. Alzheimer's Research \& Therapy. 7: 50.

[10]. Kalpakliglu AF, Kalkan IK, Akcay A, Reisli I, Can D, et al. (2011). Un (awareness) of Allergy. World Allergy Organization Journal. 11; 171-178.

[11]. Management of Allergic rhinitis and its impact on asthma. Global Primary Care Education. 2007

[12]. Mandhane SN, Shah JH, Thennati R (2011). Allergic rhinitis: An update on disease, present treatments and future prospects. Int. Immunopharmacol. 1646-1662.

[13]. Marcano Belisario JS, Jamsek J, Huckvale K, O'Donoghue J, Morrison CP, \& Car J. (2015). Comparison of self-administered survey questionnaire responses collected using mobile apps versus other methods. Cochrane Database of Systematic Reviews, Issue 7. Art. No.: MR000042.

[14]. Marshall PS, O'Hara C, Steinberg P. (2002). Effects of seasonal allergic rhinitis on fatigue levels and mood. Psychosom Med. 64(4); 684-691.

[15]. Nulty DD (2008). The adequacy of response rates to online and paper surveys: what can be done? Assessment \& Evaluation in Higher Education. 33(3): 301-314.

[16]. Prasad R, \& Kumar R (2013). Allergy Situation in India: What is Being Done? Indian J Chest Dis Allied Sci. $55: 7-8$

[17]. Sansone RA, Sansone LA (2011). Allergic rhinitis: relationships with anxiety and mood syndromes. Innov Clin Neurosci. 2011; 8(7): 12-17.

[18]. Scadding GK, Durham SR, Mirakian R, Jones NS, Leech SC, Farooque S, et al (2008). BSACI guidelines for the management of allergic and non-allergic rhinitis. Clinical and Experimental Allergy. 38, 19-42

[19]. Scarupa MD, \& Kaliner MA (2015) In-Depth Review of Allergic Rhinitis. World Allergy Organization. Available from:

http://www.worldallergy.org/professional/allergic_diseases_center/rhinitis/rhinitis_indepth.php (Accessed on 01 September, 2016)

[20]. Shah A, \& Pawankar R (2009). Allergic Rhinitis and Co-morbid Asthma: Perspective from India- ARIA Asia-Pacific Workshop Report. Asia Pacific Journal of Allergy and Immunology. 27:71-77.

[21]. Skoner DP. (2001) Allergic rhinitis: definition, epidemiology, pathophysiology, detection, and diagnosis. J Allergy Clin Immunol. 108(1 Suppl):S2-8.

[22]. Smith SK, Selig W, Harker M, Roberts JN, Hesterlee S, Leventhal D, et al. (2015) Patient Engagement Practices in Clinical Research among Patient Groups, Industry, and Academia in the United States: A Survey. PLoS ONE 10(10): e0140232.

[23]. Viswanathan R. (1964) Definition, incidence, aetiology and natural history of asthma. Indian J Chest Dis 6:108-24.

[24]. Wallis RS (1982). The problem of allergic rhinitis. Modern Medicine. 6; 29-37.

[25]. World Allergy Organization. Available from: http://www.worldallergy.org/professional/allergic_diseases_center/rhinitis/rhinitissynopsis.php (Accessed on 01 September, 2016)

[26]. World Health Organization. White Book on Allergy (2011) Executive Summary. Available from: http://www.worldallergy.org/UserFiles/file/WAO-White-Book-on-Allergy_web.pdf (Accessed on 01 September, 2016). 